\title{
Are dietitians able to estimate patient's weight accurately?
}

\author{
K. L. Barker and Acute Team Dietitians \\ North Bristol NHS Trust, Bristol Westbury-on-Trym BS10 5NB, UK
}

It has been nationally recommended that all patients are screened for malnutrition on admission to hospital and weekly thereafter ${ }^{(1)}$. In reality, the weighing of acutely unwell patients is difficult in the face of ward staff pressures and suitable equipment. In a report by BAPEN ${ }^{(2)}$, just $6 \%$ hospitals screen $75-$ $100 \%$ patients. This highlights that there are potential difficulties in obtaining the basic measurements needed to carry out malnutrition screening.

The need to improve regular weighing of patients is crucial in performing accurate Dietetic assessments. In the absence of a body weight measurement, Dietitians base weight estimations on their best clinical judgement. Nutritional treatment is often based on estimated calculations. If it is possible to establish the accuracy of weight estimations by qualified Dietitians, this would give evidence to further support the accurate measurement by ward staff, or give us confidence in our abilities to accurately estimate weight. Within our own hospital a previous audit had shown that only $40 \%$ of our in-patients had their body weight recorded. This is similar to the BAPEN ${ }^{(2)}$ survey which found that $49 \%$ hospitals record patients' weights across all wards. Previous work on estimation of body weight found that physicians were able to estimate body weight with a mean difference of approximately $5.5 \mathrm{~kg}$ and the patients own estimation gave a mean difference of $2.7 \mathrm{~kg}^{(3)}$.

Sixteen patients on an elderly acute ward were weighed by the ward staff using calibrated digital sit-down scales. Over a two-day period, eight qualified Dietitians were asked to estimate the patient's body weight. Dietitians were asked to estimate each patient's weight without knowledge of their clinical condition. Patients ranged from lying to seated position, no other equipment such as height measure or girth was used, and Dietitians did not ask the patients about their weight history. The actual and estimated weights were recorded and comparisons were drawn.

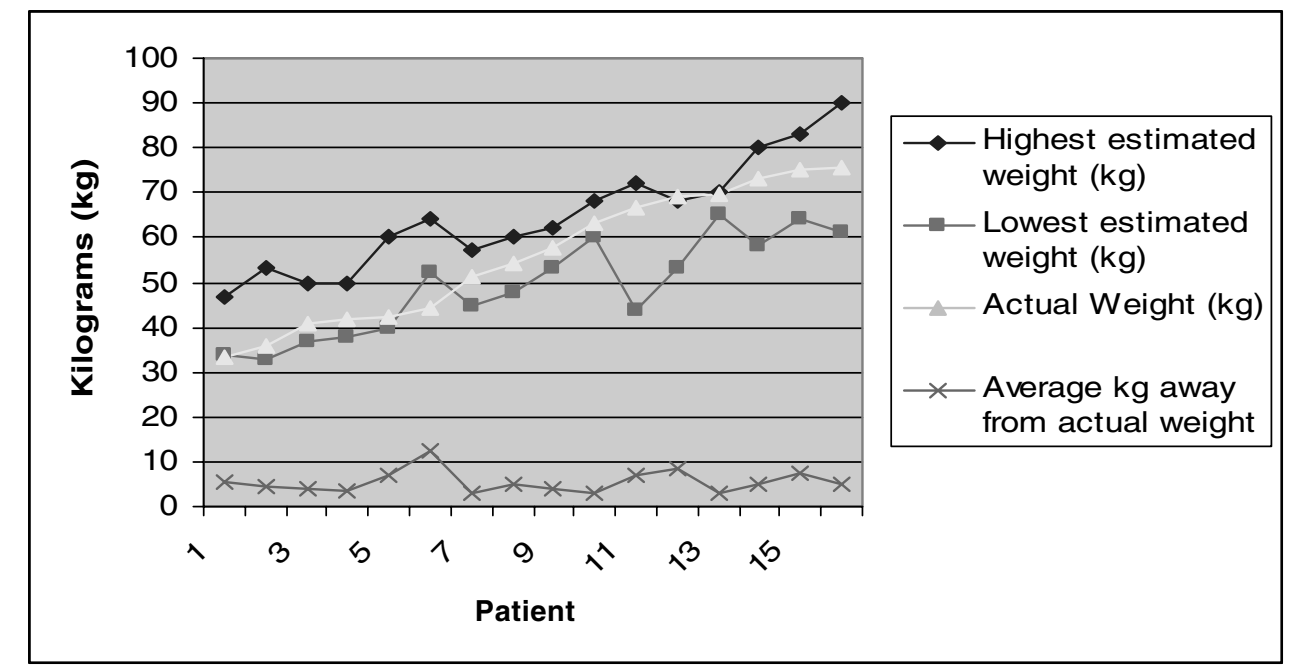

On average across all patient body weights, the estimation differed by $5 \mathrm{~kg}$. Correct weight guesses within $2 \mathrm{~kg}$ ranged from $12.5 \%$ to $50 \%$ of estimations by individual Dietitians. In total, $30 \%$ of Dietician's estimations of body weight were within $2 \mathrm{~kg}$. Those Dietitians who are primarily ward based appeared to have more correct estimations within $2 \mathrm{~kg}$.

Overall weight estimations vary on average by $5 \mathrm{~kg}$. This could lead to an additional calorie estimation or deficit of $63 \mathrm{kcal}$ for a $60 \mathrm{~kg}$ women or $81 \mathrm{kcal}$ for a $70 \mathrm{~kg} \mathrm{man}{ }^{(4)}$. This audit showed that the maximum amount of weight over-estimated was $23 \mathrm{~kg}$, and an under-estimation of $20 \mathrm{~kg}$. Based on a $70 \mathrm{~kg}$ man (aged 60-74 years) this could lead to a calorie deficit of $621 \mathrm{kcal}$ or an additional $370 \mathrm{kcal}$ to calculated requirements ${ }^{(5)}$. This could potentially lead to weight gain or loss within dietetic treatment plans.

It can be suggested that Dietitians are very unlikely to be able to identify patient's body weight changes over time. The recommendations for malnutrition screening include repeated assessment to identify small weight changes. First line treatment advice is based on the ability to identify unintentional weight loss and without this assessment in place a screening tool may become invalid.

Problems that hinder regular weighing of patients in hospital include accurate calibration of ward equipment and dedicated ward time. The audit has shown that Dietitians are unable to accurately predict patients' body weight within $2 \mathrm{~kg}$ in no more that $30 \%$ of patients, and from this they are highly unlikely to identify changes in body weight and monitor dietetic plans effectively. This highlights the importance of obtaining an accurate body weight measurement and the barriers to weighing patients need to be identified and tackled.

1. National Institute for Health and Clinical Excellence (NICE) (2006) Nutrition Support in Adults, Clinical Guideline 32. London: NICE.

2. Russel CA, Elia M, on behalf of BAPEN and Collaborators (2007) Nutrition Screening Survey in the UK in 2007. RCN: Enhancing Nutritional Care.

3. Lorenz MW, Graf M, Henke C et al. (2007) JNeurol Neurosurg Psychiatry 78, 1331-1336.

4. RCN Guidelines Enhancing Nutritional Care: Publication 003284

5. Schofield WN (1985) Hum Nutr Clin Nutr 39c, Suppl. 1, 5-41. 